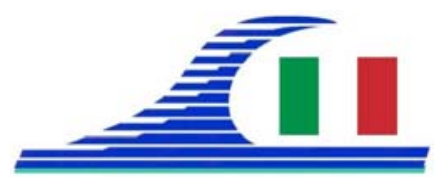

Conférence Méditerranéenne Côtière et Maritime EDITION 3, FERRARA, ITALIA (2015)

Coastal and Maritime Mediterranean Conference

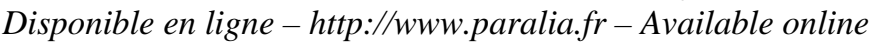

\title{
Caractérisation des sédiments fins des retenues hydroélectriques en vue d'une pré-orientation vers des filières de valorisation matière
}

\author{
Baptiste ANGER ${ }^{1}$, François THERY ${ }^{1}$, Daniel LEVACHER ${ }^{2}$
}

1. EDF R\&D, Département Eco-efficacité et Procédés Industriels (EPI), EDF Lab. Les Renardières, 77818 Moret-sur-Loing, France.

francois.thery@edf.fr

2. UMR CNRS 6143 Morphodynamique Continentale et Côtière (M2C), 24 rue des Tilleuls, 14000 Caen, France.

\section{Résumé :}

Electricité de France (EDF) exploite une large part des installations de l'ensemble du parc hydraulique français. A ce titre elle doit gérer le transit sédimentaire sur ses installations sachant qu'une partie des sédiments peut y être retenue. La question de la gestion à terre de ces sédiments est parfois abordée, le manque de retour d'expériences positives sur les voies de valorisation à terre des sédiments fins de dragage marins et fluviaux révèle l'absence de solutions durables. EDF a donc souhaité travailler à l'élaboration d'un outil de pré-orientation à partir d'une proposition de caractérisation en accord avec les spécifications techniques des filières potentielles, à savoir, en cimenterie, en intégration dans le béton, en matériau routier et assimilé, en matériau céramique et en valorisation agronomique. L'outil proposé est original et apporte aujourd'hui à l'exploitant des installations hydrauliques une première détermination des filières qui doivent être étudiées pour une valorisation définitive.

Mots-clés : Sédiments fins, Ressource minérale alternative, Valorisation matière, Economie circulaire.

\section{Introduction}

Les opérations de dragage de sédiments sont nécessaires pour garantir une bonne exploitation des ports, des voies navigables et des retenues d'eau pour la navigabilité ou pour la production d'énergie.

En France, 622 barrages et 435 centrales permettent à EDF de produire approximativement 10 \% de son électricité à partir de l'énergie hydraulique. En 2013, la production nette en France métropolitaine est de 42,6 TWh à partir du parc de production hydraulique d'EDF.

Les retenues hydroélectriques sont soumises à des dépôts de sédiments ayant des impacts variables selon le bassin versant, la taille et la conception de la retenue. Dans le cadre de la gestion des dépôts sédimentaires, des impératifs techniques ou environnementaux peuvent contraindre les opérateurs à s’écarter des solutions de 
Côtes méditerranéennes menacées :

Risques et défis dans le contexte du changement climatique

gestion usuelles consistant à réintégrer les sédiments dans le cours d'eau et envisager une gestion à terre.

La valorisation des sédiments fins constitue alors un défi en matière (i) de maintien d'un niveau satisfaisant de la fonctionnalité des aménagements et (ii) de préservation de ressources naturelles.

Le peu de retour d'expériences positives sur les voies de valorisation à terre des sédiments fins de dragage marins et fluviaux révèle l'absence de solutions durables. La gestion à terre des sédiments fins de retenues hydroélectriques nécessite une vision intégratrice de l'ensemble des potentialités de valorisation sur le territoire.

Face à ces constats, l'objectif majeur est de faciliter la décision des exploitants confrontés à la gestion des sédiments. Cela s'est traduit par (i) la proposition d'une caractérisation en accord avec les spécifications techniques des filières potentielles et (ii) l'élaboration d'un outil de pré-orientation (ANGER, 2014).

\section{Démarche globale}

Pour répondre à cet objectif, un examen de cinq voies de valorisation potentielles, à savoir, en cimenterie, en intégration dans le béton, en matériau routier et assimilé, en matériau céramique et en valorisation agronomique, a été mené avec la mise en évidence des spécifications d'entrée dans le cas des sédiments fins de barrage, la recherche des paramètres clés, consolidant un inventaire des travaux de recherche et applications dans chaque filière. Cette phase permet de connaitre les caractéristiques pour lesquelles les sédiments seraient éligibles aux voies de valorisation.

La plupart des travaux portant sur la valorisation des sédiments ne concerne qu'une seule filière (AOUAD et al., 2012 ; LIMEIRA et al., 2011 ; DUBOIS et al., 2009 ; MEZENCEVOVA et al., 2012 ; SHEEHAN et al., 2010)

Dans le cadre des présents travaux, ces filières ont été simultanément mises en œuvre à l'échelle du laboratoire pour quatre sédiments fins provenant de quatre retenues hydroélectriques, représentatifs de différentes typologies. Tout d'abord une caractérisation minérale, géotechnique et agronomique a permis de discuter de l'adéquation sédiments - filières au regard des critères d'entrée dans les voies de valorisation. S'ensuivent les essais de valorisation i.e. fabrication d'un clinker, addition de sédiments dans un mortier (ANGER et al., 2014), élaboration d'un matériau routier et confection d'un matériau céramique (ANGER et al., 2013 ; GOURE-DOUBI et al., 2014), mettant en avant les caractéristiques favorables ou défavorables des sédiments fins en relation avec la filière étudiée.

Dans cette démarche globale, une hypothèse de travail a été adoptée : l'intérêt est prioritairement donné à l'étude de la caractérisation de la fraction valorisable avant une phase d'identification des polluants freinant les possibilités de valorisation. 
Mediterranean coasts at threat:

Hazards and challenges in the context of climate variability

\section{Résultats des essais en laboratoire}

Les quatre sédiments fins de barrage échantillonnés présentent les caractéristiques générales suivantes :

- Classes granulométriques des limons et des argiles (d50 de 6,1 à 37,7 $\mu \mathrm{m}$ ) ;

- Principaux minéraux : quartz (24 à 36 \%), carbonates (4 à $24 \%$ ) et cortège de minéraux argileux (22 à $35 \%$ ) dominé par des familles des illites et chlorites ;

- Teneur en matière organique (MO) variant de 3 à $9 \%$ selon les cas étudiés.

Concernant la mise en œuvre de quatre applications potentielles à l'échelle du laboratoire, les principaux résultats sont les suivants :

- Des clinkers ont été fabriqués à partir de crus comprenant 20 à $30 \%$ de sédiment et sont similaires à un clinker Portland ordinaire du point des compositions chimiques et minéralogiques.

- Les mortiers testés intégrant des sédiments comme addition minérale ne permettent pas d'obtenir des performances équivalentes aux additions normalisées de type calcaire ou siliceux. On peut retenir cependant qu'une incorporation de sédiments fins de barrage dans certains mortiers est envisageable ;

- Des performances encourageantes (Rc>1 MPa) ont été obtenues avec un traitement type chaux/ciment permettant d'envisager une utilisation comme matériau routier.

- Des pièces céramiques de type terre cuite ont été confectionnées à base de sédiments avec ou sans apport d'autre matière argileuse.

Si les résultats des essais de valorisation confirment qu'il est possible d'utiliser ces sédiments fins de barrage dans plusieurs applications, il n'en demeure pas moins que le niveau d'aptitude à leur emploi dans telle ou telle filière, est fortement conditionné par leurs caractéristiques.

\section{Adéquation sédiments-filières}

Un cadre méthodologique a été développé aboutissant à un outil aidant à pré-orienter les sédiments fins vers les voies de valorisation adaptées. La démarche s’articule en trois étapes, voir figure 1. Ce cadre méthodologique a été proposé en s'appuyant sur les travaux menés par ANDREWS et al. (2004) ayant trait à la qualité des sols.

Les critères indicateurs sont sélectionnés selon les spécifications d'entrée des applications et/ou pour permettre la comparaison entre les caractéristiques des sédiments et des matières premières traditionnelles. Selon l'application, 3 à 7 critères sont sélectionnés.

Dans l'outil, chaque critère indicateur est transformé par l'intermédiaire d'une courbe de score en une note sans unité comprise entre 0 et 1 ; 1 signifiant que le critère est parfaitement dans la gamme de valeur attendue.

Les scores sans unité sont ensuite combinés pour former une seule valeur, appelée indice d'adéquation (IA), en faisant une moyenne pondérée des scores puis en la multipliant par 10 (voir fig. 1, étape 3). 
Côtes méditerranéennes menacées :

Risques et défis dans le contexte du changement climatique

Pour chaque application considérée, le critère indicateur (par exemple le d50, la teneur en $\mathrm{MO}$, les limites d'Atterberg, ...) est interprété et pondéré en tenant compte de la bibliographie, intégrant la littérature scientifique, les normes, les guides techniques, ainsi que les expériences effectuées en laboratoire et la prise en considération d'avis d'experts.

Les IA permettent finalement d'identifier, à l'aide de notes, l'aptitude technique d'utilisation des sédiments dans les filières de valorisation.

Une description complète de cet outil pour évaluer l'adéquation technique entre les caractéristiques des sédiments et les spécifications des filières de valorisation est proposée par ANGER (2014).

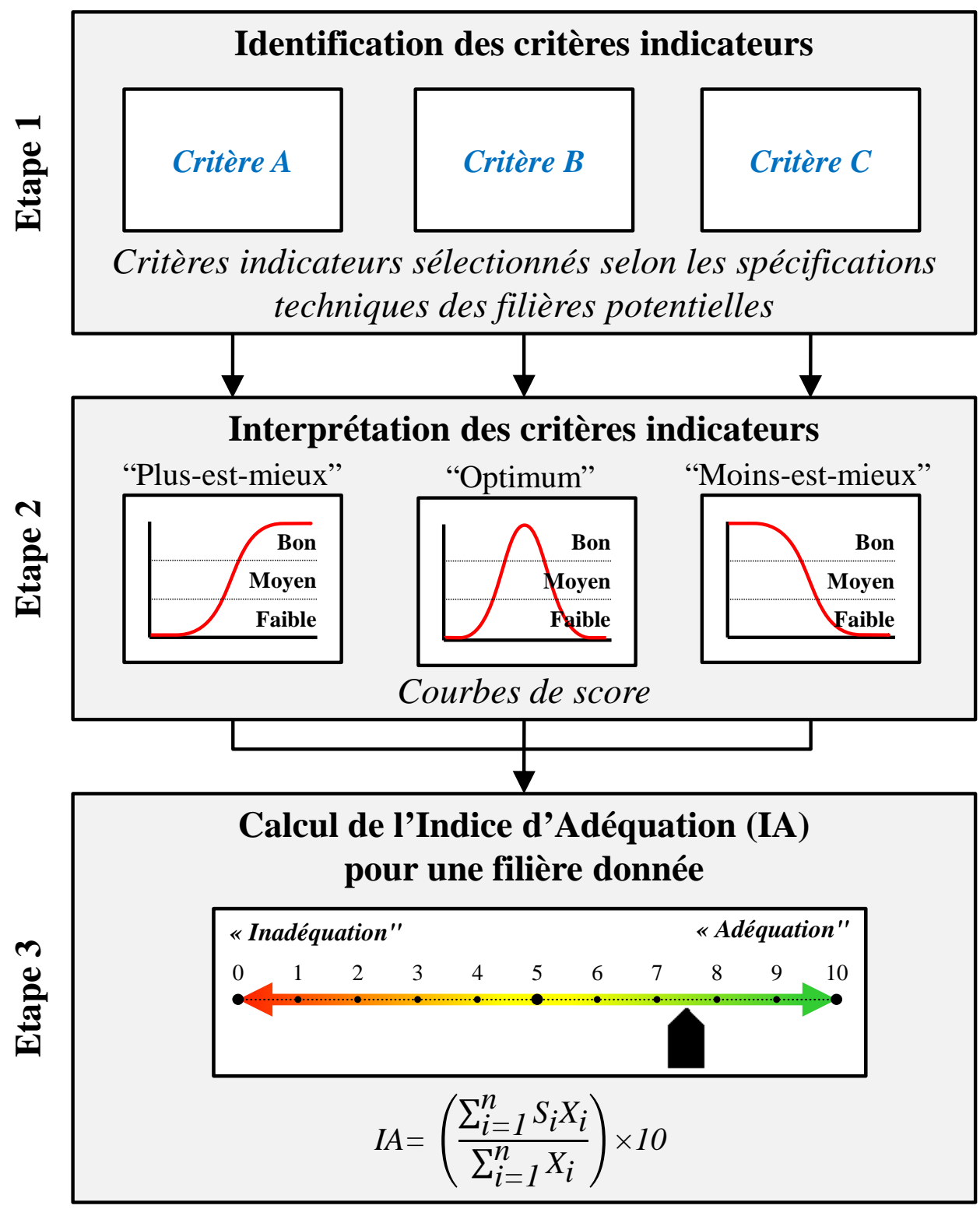

Figure 1. Schéma conceptuel de l'outil. 
Pour utiliser cet outil de pré-orientation, il est nécessaire de disposer au préalable d'un jeu de données minimum caractérisant les sédiments fins dont la valorisation est envisagée. A cet effet, une caractérisation a été proposée. Elle regroupe les essais nécessaires pour disposer d'un ensemble de données minimum et les paramètres techniques qu'il convient de connaitre si une gestion à terre des sédiments fins est envisagée.

En utilisant l'outil pour les 4 sédiments étudiés, il apparait que les filières dans lesquelles l'utilisation de sédiments fins de barrage est techniquement la plus adaptée, concernent l'utilisation des sédiments fins en matière première en cru de cimenterie (IA moyen=8,85/10) et la fabrication de céramique de type terre cuite (IA moyen=7,85/10). Pour les autres applications, la variabilité des résultats entre les différentes caractéristiques des sédiments ne permet pas de conclure de façon générale et oblige à traiter au cas par cas.

Comme ces deux filières impliquent fortement la prise en compte du tissu industriel, cet aspect a été considéré et a donné lieu à un travail spécifique consistant à identifier géographiquement les sites d'accueil où les sédiments fins peuvent être valorisés, (ANGER, 2014).

Ce travail contribue aussi à mettre en avant des opportunités locales de valorisation et à mettre en relief les synergies potentielles entre les acteurs possédant les gisements de sédiments fins et les acteurs pouvant utiliser ces matériaux alternatifs. Toutefois, la puissance des gisements n’a pas encore été étudiée.

\section{Conclusion et perspectives}

Une approche durable en matière de gestion des matériaux de dragage exige notamment un changement de point de vue. Ces travaux qui contribuent à l'amélioration de la connaissance des atouts et contraintes de ces sédiments fins et des voies de valorisation, tendent à bâtir des filières robustes.

Bien que les sédiments gérés à terre aient un statut réglementaire de déchet, ils méritent d'être considérés comme une ressource alternative potentielle. De plus, leur réutilisation correspond parfaitement à la philosophie d'une économie circulaire.

En proposant une approche globale adaptée aux sédiments de barrage, ces travaux ont abouti à la mise en œuvre d'un outil décisionnel de pré-orientation des sédiments vers des filières de valorisation les plus adaptées.

A ce jour, l’outil a été rendu opérationnel et est utilisé sur d'autres bassins versants, ce qui devrait apporter à moyen terme suffisamment de retour d'expériences pour permettre de l'enrichir par diverses typologies de sédiments.

L'équipe d'EDF R\&D qui a participé à la réalisation de ces travaux et à la mise en œuvre de la démarche, travaille aujourd'hui à la détermination de l'impact de certains polluants contenus dans les sédiments sur les filières retenues. La mise en place d’une 
Côtes méditerranéennes menacées :

Risques et défis dans le contexte du changement climatique

base de données dite géographique afin de croiser les positions des ouvrages sur le territoire avec les filières de valorisation est en cours de développement.

\section{Références bibliographiques}

ANDREWS S.S., KARLEN D.L., CAMBARDELLA C.A. (2004). The soil management assessment framework: A quantitative soil quality evaluation method. Soil Sc. Society of America Journal, Vol. 68, pp 1945-1962. http://dx.doi.org/10.2136/sssaj2004.1945 ANGER B. (2014). Caractérisation de sédiments fins de retenues hydroélectriques en vue d'une orientation vers des filières de valorisation matière. Thèse de doctorat, Université de Caen Basse-Normandie, 309 p.

ANGER B., LECOMTE-NANA G., PEYRATOUT C., THERY F., LEVACHER D. (2013). Retenues hydroélectriques : source renouvelable de matières premières pour les céramiques silicatées ? Industrie Céramique et Verrière, $n^{\circ} 1047$, novembre/décembre 2013, pp 40-45.

ANGER B., MOULIN I., PERIN E., THERY F., LEVACHER D. (2014). Utilisation de sédiments fins de barrage dans la fabrication de mortiers. XIII ${ }^{\text {èmes }}$ Journées Nationales Génie Côtier - Génie Civil, Dunkerque, pp 953-960. http://dx.doi.org/10.5150/ingcgc.2014.105

AOUAD G., LABOUDIGUE A., GINEYS N., ABRIAK N.E. (2012). Dredged sediments used as novel supply of raw material to produce Portland cement clinker. Cement and Concrete Composites, Vol. 34(6), pp 788-793. http://dx.doi.org/10.1016/j.cemconcomp.2012.02.008

DUBOIS V., ABRIAK N.E., ZENTAR R., BALLIVY G. (2009). The use of marine sediments as a pavement base material. Waste Management, Vol. 29(2), pp 774-782. http://dx.doi.org/10.1016/j.wasman.2008.05.004

GOURE-DOUBI H., LECOMTE-NANA G., THERY F., PEYRATOUT C., ANGER B., LEVACHER D. (2015). Characterization and valorization of dam sediment as ceramic materials. International Journal of Engineering and Innovative Technology, Vol. 4(8), pp 8491.

LIMEIRA J., ETXEBERRIA M., AGULLO L., MOLINA D. (2011). Mechanical and durability properties of concrete made with dredged marine sand. Construction and Building Materials, Vol. 25(11), pp 4165-4174. http://dx.doi.org/10.1016/j.conbuildmat.2011.04.053 MEZENCEVOVA A., YEBOAH N.N., BURNS S.E., KAHN L.F., KURTIS K.E. (2012). Utilization of Savannah Harbor river sediment as the primary raw material in production of fired brick. Journal of Environmental Management, Vol. 113, pp 128-136. http://dx.doi.org/10.1016/i.jenvman.2012.08.030

SHEEHAN C., HARRINGTON J., MURPHY J. (2010). A technical assessment of topsoil production from dredged material. Resources, Conservation and Recycling, Vol. 54(14), pp 1377-1385. http://dx.doi.org/10.1016/j.resconrec.2010.05.012 